\title{
BMJ Open Management in the paediatric wards facing novel coronavirus infection: a rapid review of guidelines and consensuses
}

\author{
Wen-Yi Luo, ${ }^{1,2}$ Ji-Wen Sun, ${ }^{1}$ Wen-Lan Zhang, ${ }^{3}$ Qian Li, ${ }^{4}$ Ping Ni, ${ }^{1}$ Lie-Bin Zhao (D , ${ }^{5}$ \\ Jin-Hui Tian, ${ }^{6}$ Ya-Qing Zhang, ${ }^{7}$ Hong Lu ${ }^{1}$
}

To cite: Luo W-Y, Sun J-W, Zhang W-L, et al. Management in the paediatric wards facing novel coronavirus infection: a rapid review of guidelines and consensuses. BMJ Open 2020;10:e039897. doi:10.1136/ bmjopen-2020-039897

- Prepublication history for this paper is available online To view these files, please visit the journal online (http://dx.doi org/10.1136/bmjopen-2020039897).

W-YL, J-WS and W-LZ contributed equally.

Received 29 April 2020 Revised 27 July 2020 Accepted 30 July 2020
Check for updates

(C) Author(s) (or their employer(s)) 2020. Re-use permitted under CC BY-NC. No commercial re-use. See rights and permissions. Published by BMJ.

For numbered affiliations see end of article.

Correspondence to Dr Lie-Bin Zhao; zhaoliebin@163.com and Professor Ya-Qing Zhang; zhangyqf@163.com

\section{ABSTRACT}

Objectives Relevant guidelines and consensuses for COVID-19 contain recommendations aimed at optimising the management in paediatric wards. The goal of this study was to determine the quality of those recommendations and provide suggestions to hospital managers for the adjustment of existing hospital prevention and control strategies, and also to offer recommendations for further research.

Design A rapid review of the guidelines and consensuses for the management in paediatric wards facing COVID-19.

Methods PubMed, EMBASE, the Cochrane Library, UpToDate, China National Knowledge Infrastructure, the Wanfang database and relevant websites such as medlive. $\mathrm{cn}, \mathrm{dxy} . \mathrm{cn}$, the National Health and Health Commission and the China Center for Disease Control and Prevention were systematically searched through late May 2020. The Appraisal of Guidelines for Research and Evaluation II (AGREE II) tool was then used to assess the quality of the selected articles and summarise the relevant evidence concerning management in paediatric wards.

Results A total of 35 articles were included, composed of 3 consensus guidelines, 25 expert consensuses and 7 expert opinions. Of the 35 papers, 24 were from China, 2 from the USA, 1 from Spain, 1 from Brazil, 1 from Saudi Arabia and 6 from multinational cooperative studies. Scores for the six domains of the AGREE II tool (scope and purpose, stakeholder involvement, rigour of development, clarity of presentation, applicability and editorial independence) were $98.57 \%, 53.57 \%, 17.92 \%, 69.62 \%$, $26.96 \%$ and $50.35 \%$, respectively. Recommendations for nosocomial infection and control, human resource management as well as management of paediatric patients and their families were summarised.

Conclusions Due to the outbreak of COVID-19, the quality of rapid guidelines and consensuses for the management in paediatric wards affected by COVID-19 is unsatisfactory. In the future, it will be necessary to develop more highquality guidelines or consensuses for the management in paediatric wards to deal with nosocomial outbreaks in order to fully prepare for emergency medical and health problems.

\section{Strengths and limitations of this study}

- This rapid review of guidance focused on management in paediatric wards affected by COVID-19 will help paediatric healthcare providers take action in their wards.

- We employed the Appraisal of Guidelines for Research and Evaluation II tool, a scientifically valid instrument used to assess article quality, to summarise all key recommendations, after which we compared the inconsistencies among them.

- We included documents published in Chinese or English only, thereby running the risk of neglecting essential documents from regions in which Chinese or English is not the first language.

- Unconscious bias from a subjective rating of documents was inevitable. Therefore, we avoided inviting coauthors of the guidance documents to be reviewers in order to prevent subconscious competing interests, and we conducted two rounds of group discussions to minimise subjective bias.

\section{INTRODUCTION}

On 30 January 2020, the WHO announced that the COVID-19 epidemic was a public health emergency of international concern. ${ }^{1}$ The COVID-19 pandemic is developing rapidly. According to the latest $\mathrm{WHO}$ data, as of 10:00 China Standard Time on 26 July 2020, there were 15785641 confirmed cases of COVID-19 and 640016 deaths worldwide, involving 215 countries or regions. ${ }^{2}$

In China, the Law on the Prevention and Treatment of Infectious Diseases divides such diseases into three categories in descending order of severity. COVID-19 has been categorised into category B (which covers nearly 30 diseases, including SARS and COVID-19), although prevention and control have been carried out in accordance with category A (which covers only bubonic plague and cholera) guidelines. National-level policy specifications such as diagnosis, treatment 
plans and protection guidelines have been issued, which provide a basis for the standardised clinical diagnosis and control of COVID-19. In the current reported cases of the COVID-19 pandemic, patients of all ages have been infected. Studies have already reported COVID-19 infection in children. ${ }^{3}$ Therefore, paediatric professional groups and paediatric experts in all municipalities have formulated guidelines and consensuses on the topic of paediatric COVID-19, also involving the diagnosis and treatment of diseases in general, and the prevention and control of nosocomial infections.

During the process of COVID-19 prevention and control, standardisation of the management for the entire paediatric ward is important. This requires the implementation of reasonable standardised and humane management strategies designed to provide managers with the ability to take appropriate actions in order to deal with major emergencies, and to offer acceptable methods to care for children with suspected and confirmed cases, as well as their families, especially vulnerable neonates. These are the challenges and existing problems for clinical staffs facing the outbreak.

Therefore, this study was conducted to summarise and analyse the published articles concerning the management in paediatric wards affected by COVID-19, compare any differences and provide recommendations to paediatric medical staffs and hospital managers for the adjustment of existing hospital prevention and control strategies.

\section{METHOD}

\section{Literature search and search strategies}

A systematic search (last updated before 31 May 2020) was conducted to retrieve relevant guidelines and consensuses concerning the management in paediatric wards facing COVID-19. The major academic databases included PubMed, EMBASE, the Cochrane Library, UpToDate, China National Knowledge Infrastructure, the Wanfang database and relevant websites such as medline.cn, dxy. cn, the National Health and Health Commission and the China Center for Disease Control and Prevention, each of which was searched systematically. Search terms included novel coronavirus, coronavirus pneumonia, COVID, COVID-19, NCP, nCoV, guideline, consensus, recommendation, opinion, pediatrics, children, newborn, and others. The search strategies are listed in online supplementary appendix 1 .

\section{Inclusion and exclusion criteria}

The inclusion criteria included: (1) the target group of the article was patients with COVID-19; (2) guidelines, consensuses, recommendations or expert opinions issued by international or national/regional organisations or associations; (3) inpatient wards, isolation ward management, childcare and personnel management; (4) latest version; (5) published in Chinese or English; and (6) full text available. The exclusion criteria included: (1) interpretation version; (2) translated version; (3) literature type was a research plan or proposal. Two reviewers (WZ and QL) independently screened all searched articles. Disagreements between the reviewers were resolved via face-to-face discussion with a third reviewer $(\mathrm{PN})$.

\section{Data extraction}

All of the included studies were imported into the EndNote software platform. Two reviewers (WL and JS) independently extracted and cross-checked relevant data (article characteristics, recommendations for management) from each included article. Data extraction was conducted in Microsoft Excel. In order to ensure data validity and accuracy, the data collection was completed by two researchers independently. Disagreements were resolved by discussion.

\section{Quality assessment}

The 23-item Appraisal of Guidelines for Research and Evaluation II (AGREE II) tool ${ }^{4}$ across six domains (scope and purpose, stakeholder involvement, rigour of development, clarity of presentation, applicability and editorial independence) was used by two researchers (WZ and QL) to independently evaluate the quality of each article. Each item was assigned a score ranging from 1 (strongly disagree) to 7 (strongly agree) points. The score for each domain was calculated by summing all scores of the individual items in a domain and then standardised as a percentage using the following equation: domain score $=($ obtained score - minimal possible score $)$ / (maximal possible score-minimal possible score). ${ }^{5}$ The overall assessment included whether the articles could be recommended for clinical practice. ${ }^{5}$ An overall assessment of each article is included in the AGREE II tool: if the score is $>60 \%$, the grade is 'strongly recommended for use in practice'; if the score is between $30 \%$ and $60 \%$, the grade is 'recommended for use with some modification'; if the score is $<30 \%$, the grade is 'not recommended for use in practice'. ${ }^{6}$

\section{Patient and public involvement}

Patients were not involved in this research.

\section{Data analysis}

The means and SDs for the AGREE II domains were calculated and presented. The intraclass correlation coefficients (ICCs) were used for testing inter-rater reliability among researchers. ${ }^{7}$ ICC values from 0.81 to 1.00 were considered to be the most consistent. ${ }^{8}$ SPSS V.22.0 software was used for statistical analysis.

\section{RESULTS}

\section{Search results}

Our systematic search yielded a total of 792 records after duplicates were removed, and 738 were excluded due to irrelevant issues after screening by title and abstract. After assessment of the full text, 19 of the remainder were excluded, leaving a total of 35 articles $^{9-43}$ that met the 
selection criteria. The screening process and results are illustrated in figure 1.

\section{Characteristics of included articles}

The 35 selected studies were conducted by various medical organisations and published in the year 2020 . These articles were composed of 3 sets of consensus guidelines, 25 expert consensuses and 7 expert opinions. Thirteen were written in English, 23 in Chinese. Of the total 35 papers, 24 were from China, 2 from the USA, 1 from Spain, 1 from Brazil, 1 from Saudi Arabia and 6 from multinational cooperative efforts. Five were second editions, while the rest were first editions. The number of participating centres ranged from 1 to 57 . The results are listed in online supplementary appendix 2.

\section{Quality scores}

The ICC for AGREE II was 0.898 (95\% CI 0.884 to 0.911 ), indicating that the consistency was excellent. See online supplementary appendix 2 for the results.

\section{Content summary and analysis \\ Nosocomial infection and control}

There were 13 recommendations for nosocomial infection and control, concerning ward setting and personal protection management. In the personal protection management, there are three levels of protection. Level I protection: uniform, medical/surgical mask, hand hygiene, disposable latex gloves, disposable hats and isolation clothing (level I+). Level II protection: work clothes, a medical protective mask (N95), hand hygiene, disposable latex gloves, a disposable hat, goggles or a protective mask (level II+), isolation clothing, medical protective clothing (level II + ) and shoe covers. Level III protection: work clothes, a medical protective mask (N95), hand hygiene, disposable latex gloves, a disposable hat, goggles or a protective mask, isolation clothing, medical protective clothing, shoe covers and (when necessary) comprehensive respiratory protectors. All the recommendations came from 25 articles of varying quality. A summary of these recommendations is provided in online supplementary appendix 3 .

\section{Isolation ward setting}

1. In the isolation ward setting, a child suspected of having COVID-19 needs a single-occupant room. $^{10-121415192027-293133}$

2. Diagnosed children can stay in the same room. ${ }^{10-12} 1415202128293133$

3. Critically ill children should be sent to a negative pressure room in the intensive care unit (ICU). ${ }^{11-1316282936}$

4. If a newborn or premature infant's mother is suspected of having or suffering from COVID-19, the infant should be transferred to an isolation room. ${ }^{15333538}$

5. Incubators are recommended as isolation tools for newborns. 14152738
Isolation ward: personal protection management

6 . For personal protection management in the isolation ward, at least level II protection ${ }^{10} 14172021$ 25-27 32333538 or perhaps level III protection ${ }^{915} 19343638$ is required.

\section{General ward setting}

7. In the general ward setting, a transitional ward could be set up for patients. 152127283032

8. A single-occupant room is needed for children in the haematology and oncology departments. ${ }^{17}$

\section{General ward: personal protection management}

9. For personal protection management in the general ward, level I protection is basically required. $.^{17} 19-2132$

10. Level II protection is required in special departments, such as the infectious disease department ${ }^{10}$ and the in-centre haemodialysis department. ${ }^{34}$

\section{Special procedures: personal protection management}

11. For personal protection management during special procedures that could produce aerosols, no less than level II protection ${ }^{1014} 202538$ or perhaps level III protection $^{15172127323536384041}$ is required.

12. For the disposal of disposable medical waste, chlorine-containing solution should be used to spray the outside of waste bags, ${ }^{14-16} 20$ and disinfection should be performed for $>10 \min ^{14-1620}$ before transport.

13. Non-disposable medical fabrics should be sprayed with chlorine-containing disinfectant solution for $>10$ min before transport. ${ }^{14-16}$

\section{Human resource management}

There were four recommendations for human resource management in wards, which were derived from 11 articles of varying quality. A summary of the recommendations is tabulated in online supplementary appendix 4 .

1. Medical staff should be provided to isolation wards according to national standards ${ }^{14} 26$ or to the severity of patients' conditions ${ }^{31}$ to ensure adequate human resources. $^{34}$

2. Rotation of medical staff in isolation wards every half month ${ }^{2631}$ or 1 month $^{20}$ is needed to ensure adequate rest for staff. ${ }^{34}$

3. All medical staff should be trained in the latest knowledge of COVID-19, ${ }^{34}$ disease-related nosocomial infection prevention and control, ${ }^{1421} 26$ and psychological crisis intervention. ${ }^{20}$ In addition, simulation training specific to managing suspected COVID-19 cases should be in place in every suite. ${ }^{38}$

4. Psychological counselling should be provided for staff in fever clinics and isolation observation wards by counsellors, social workers, volunteers or professional societies. ${ }^{172143}$

\section{Management of paediatric patients and their families}

There were 12 recommendations for the management of paediatric patients and their families in hospital wards, which were derived from 26 articles of varying quality. A 

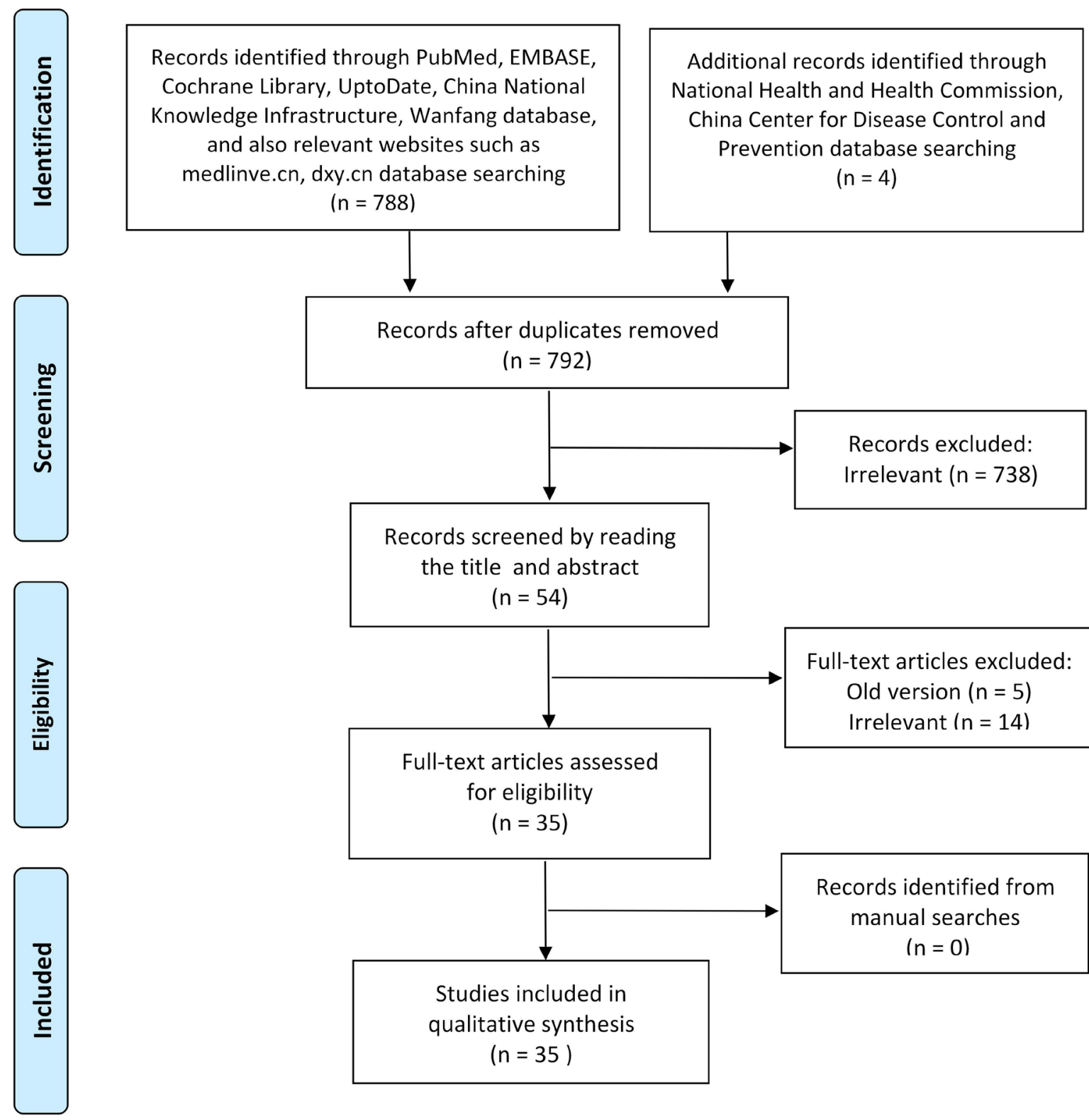

Full-text articles assessed for eligibility $(n=35)$

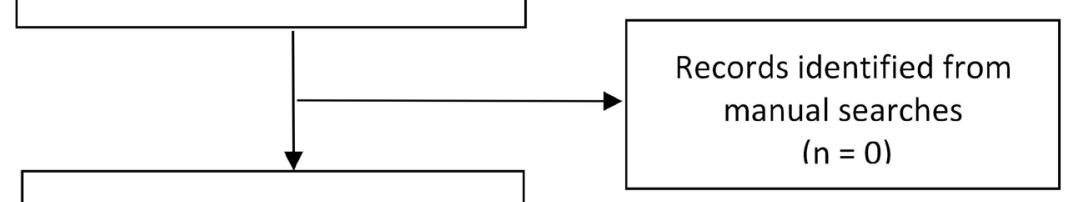

Studies included in qualitative synthesis

$$
(n=35)
$$

From: Moher D, Liberati A, Tetzlaff J, Altman DG, The PRISMA Group (2009). Preferred Reporting /tems for Systematic Reviews and MetaAnalyses: The PRISMA Statement. PLoS Med 6(7): e1000097. doi:10.1371/journal.pmed1000097

For more information, visit www.prisma-statement.org.

Figure 1 Flow chart of the literature research. Adapted from Moher et al. ${ }^{55}$ 
summary of the recommendations is tabulated in online supplementary appendix 5 .

\section{Chaperones}

1. In the general ward, chaperones should be limited to one person for each paediatric patient, ${ }^{17} 21$ and they should be required to wear masks and have their temperatures monitored. ${ }^{17213031}$

2. It is inconclusive as to whether chaperones should be permitted in the isolation ward ${ }^{21263138}$ if specifically needed, and whether there should be only one chaperone who is taking protective measures. ${ }^{19} 2026293134$

3. In the neonatal ward, chaperones are not allowed to leave the neonatal ward. ${ }^{28}$

\section{Visitors}

4. Visitors are not allowed in the neonatal ward, ${ }^{141527283132}$ isolation ward, ${ }^{16} 202126293138$ haematology and oncology ward ${ }^{17}$ or transitional ward $^{21}$ unless specifically needed. 262829

5. If the patient is in critical condition, the visitor must enter via the designated route and take strict protective measures before entering. ${ }^{26}$

6. For non-COVID-19 mother-infant dyads, mothers of neonates in the neonatal ICU (NICU) are encouraged to visit their babies. ${ }^{42}$

7. Daily screening for respiratory symptoms and influenza syndrome is recommended for fathers and mothers visiting newborns. ${ }^{35}$

8. Video visits are recommended. ${ }^{38}$

9. Day care visits in the otolaryngology ward should be reduced. $^{37}$

\section{Breast feeding}

10. For non-COVID-19 mother-infant dyads, in which mother and infant are not separated, mothers are encouraged to breast feed directly. Otherwise, mothers should express breast milk regularly. ${ }^{31} 42$

11. For suspected or confirmed COVID-19 mother-infant dyads in the isolation room, where mother and infant are separated, direct breast feeding is avoided. ${ }^{14-16272931}$ Mothers can express breast milk and offer it to their newborns via caregivers. ${ }^{18} 3542$ In areas with a high risk of outbreaks, it is better to use breast milk after it has tested negative for COVID-19 $9^{23}$ or been pasteurised in advance. ${ }^{12} 38$ Breast feeding should cease if mothers are extremely sick, ${ }^{42}$ nor should it be performed during antiviral treatment. ${ }^{12}$

\section{Psychological counselling}

12. Psychological counselling is needed for paediatric patients and their families 11121617202324 through doctors, social workers or support consultants via telemedicine. $^{1617}$

\section{DISCUSSION}

Only 3 sets of guideline, 25 expert consensuses and 7 expert opinions concerning management in paediatric wards facing COVID-19 have been published in a mere 5 months. Based on the varying quality of the reviewed guidance documents, there are still opportunities for further enhancements of methodological rigour. The majority of the documents provided clear descriptions in the domains of 'scope and purpose' and 'clarity of presentation'. In addition, only a small proportion of the documents demonstrated potential conflicts of interest. Detailed explanations of any conflicts of interest are needed, so as to facilitate readers' judgements. ${ }^{44}$ The lowest AGREE II domain score was for 'rigor of development', which judged the strict process of guideline development or the evidence assessment method. ${ }^{45}$ In response to the sudden outbreak of COVID-19, China's provinces and cities immediately launched level I prevention and control measures in order to control the spread of the epidemic during its early stage. Since patients with suspected cases of coronavirus infection will be sent to designated medical institutions across the country, it is important and urgent for experts from hospitals to quickly generate relevant prevention and control guidance for limiting the spread of COVID-19. Given this urgency, the expert group in this study had limited time to use traditional methodological techniques. Although some methodological rigour and evidence application evaluation were not satisfactory, the proposed guidance seemed to be effective in terms of the experience of antiepidemic treatment in epidemic areas. Moreover, some of the guidance proposals were updated during the study period, and provided valuable recommendations for all paediatric medical staff and managers.

According to a previous study ${ }^{46}$ of nosocomial outbreaks of SARS in hospitals, administrative factors such as cleaning, protection, protective equipment, workload of healthcare staff and training are important in both causing and preventing nosocomial outbreaks. Currently, COVID-19 could spread via various medical activities, ${ }^{47}$ and most paediatric patients are vulnerable, especially those in the neonatal, haematology and oncology wards. It is therefore vital for the healthcare providers to focus more on the topic of management in paediatric inpatient wards in this situation. In our review, paediatric inpatient wards should operate according to the relevant local policy on the prevention and treatment of infectious diseases. For the nosocomial infection and control in our review, it was clear that the recommendations in different categories were consistent, including the set-up of isolation areas, the division of general wards and the protection requirements, as well as the disposal of medical waste. There were, however, some recommendation conflicts among the proposals for personal protection management in the isolation area and the implementation of special procedures (including aerosolgenerating procedures and the collection of respiratory samples). No less than level II protection is required in isolation wards. ${ }^{10} 14172021$ 25-2732 333538 For the same situation, however, some of the articles suggest upgrading the protection level to level III. ${ }^{915} 19343638$ In addition, 
when clinical staff conduct aerosol-generating procedures, some of the articles propose the highest level of protection, ${ }^{15} 172127323536384041$ while others suggest maintaining the original level. ${ }^{10} 14202538$ This may depend on the storage of an adequate amount of personal protective equipment and the function of a ward to cure the patients with confirmed cases of COVID-19. Although there was one low-quality consensus, the recommendations were consistent with the high-quality and mediumquality documents.

The recommendations in our review were consistent in terms of the human resource management of the ward. The ward managers need to assign medical staff to isolation wards according to national standards ${ }^{142}$ or to the severity of patients' conditions ${ }^{31}$ to ensure adequate human resources. ${ }^{34}$ Thus, to ensure adequate rest ${ }^{34}$ for staff, rotation every half month ${ }^{26} 31$ or 1 month $^{20}$ is recommended. In addition, it was suggested that the managers relay the latest knowledge of COVID- $19^{34}$ and disease-related nosocomial infection prevention and control training ${ }^{142126}$ to all medical staff. As simulation is an optimal tool for improving multidisciplinary collaboration, communication and testing of system-based resources in order to maximise safety of response to realtime events, ${ }^{48}$ simulation training specific to managing suspected COVID-19 cases $^{38}$ is recommended. At the same time, employees may experience certain pressure and anxiety due to occupational exposure, work overload, cumbersome prevention and control procedures, and other factors. ${ }^{28} 30$ Therefore, psychological counselling was recommended by high-quality ${ }^{43}$ and mediumquality ${ }^{17}$ articles for all medical staff during their employment.

In addition to the management of medical staff, the management of paediatric patients and their families is also vital in this situation. First, whether or not isolation areas should allow parents to accompany their children was not determined in this study. Four documents ${ }^{21263138}$ recommended against allowing one parent chaperone, with protective measures taken, if specifically needed. ${ }^{19} 2026293134$ Second, in the general wards, each patient could be accompanied by one parent. ${ }^{17}{ }^{21}$ Additional requirements for the parents included the wearing of medical masks and daily temperature monitoring. ${ }^{17} 213031$ It was recommended that visitors not be permitted in the isolation area ${ }^{162} 2126293138$ or in certain wards hosting vulnerable patients such as the neonatal ward, ${ }^{141527283132}$ haematology and oncology ward ${ }^{17}$ or transitional ward ${ }^{21}$ unless specifically needed. ${ }^{26} 28$ If the patient is in critical condition, the visitor must enter via the designated route and take strict protective measures before entering. ${ }^{26}$ Techniques such as video visits are recommended $^{38}$ for maintaining the safety of isolated areas while simultaneously relieving the separation anxiety of paediatric patients. In addition, visitation of neonates by their mothers was encouraged for nonCOVID-19 mother-infant dyads and for neonates in the $\mathrm{NICU}^{42}$ and daily screening for respiratory symptoms and influenza syndrome is recommended for fathers and mothers visiting newborns. ${ }^{35}$ Third, five documents in this review agreed on the breast feeding management of hospitalised children. For non-COVID-19 mother-infant dyads, in which mother and infant are not separated, mothers are encouraged to breast feed directly; otherwise, they should express breast milk regularly. ${ }^{31}{ }^{42}$ It is not clear whether the novel coronavirus can be transmitted through breast milk, and evidence is currently insufficient on the safety of breast feeding and the need for motherbaby separation. WHO guidelines recommend using freshly expressed breast milk without pasteurisation, ${ }^{49}$ and other scientific institutions and societies support this evidence. ${ }^{50-53}$ Considering that certain viruses (like cytomegalovirus and herpes simplex virus) can be transmitted by this route, ${ }^{54}$ we recommend a cautious approach in our review. For suspected or confirmed COVID-19 mother-infant dyads in the isolation room, where mother and infant are separated, direct breast feeding should be avoided. ${ }^{14-16272931}$ Mothers can express breast milk and offer it to their newborns via caregivers. ${ }^{3542}$ In areas with high risk of outbreaks, it is better to use breast milk after it has tested negative for COVID- $19^{23}$ or been pasteurised in advance. ${ }^{1238}$ Furthermore, breast feeding should cease if mothers are extremely sick, ${ }^{42}$ nor should it be performed during antiviral treatment. ${ }^{12}$

Finally, more attention should be paid to hospitalised children and their families, ${ }^{11} 121617202324$ and it is also essential to provide psychological counselling via telemedicine during the epidemic. ${ }^{16}{ }^{17}$ All of the recommendations were consistent among low, medium and high-quality documents.

\section{Strengths and limitations}

Our review has several strengths. First is the rapid review of guidance focused on management in paediatric wards being impacted by COVID-19. Hence, the results of this review will help paediatric healthcare providers take action in their wards. Second, we used the AGREE II tool, which is a scientific and valid instrument used to assess article quality, and summarised all key recommendations, after which we compared the inconsistencies among them. This review also had some limitations. We only included documents published in Chinese or English, thereby running the risk of neglecting essential documents from regions in which Chinese or English is not the first language. Moreover, unconscious bias from a subjective rating of documents was inevitable. We avoided inviting coauthors of the guidance documents as reviewers in order to prevent subconscious competing interests and conducted two rounds of group discussions to minimise subjective bias.

\section{CONCLUSIONS}

Due to the sudden and rapid spread of COVID-19, the quality of quick guidance and consensuses for management in paediatric wards is not high. In the future, it 
will be necessary to systematically formulate high-quality guidelines and consensuses for paediatric ward management based on COVID-19. Complete hospital preparedness is critical for the early detection and management of public health emergencies. This will help to enhance a hospital's capacity for emergency response, improve the quality of medical care and minimise the directionless output of manpower and equipment, and even loss of life.

\section{Author affiliations}

${ }^{1}$ Department of Nursing, Shanghai Children's Medical Center Affiliated to Shanghai Jiao Tong University School of Medicine, Shanghai, China

${ }^{2}$ School of Nursing, Shanghai Jiao Tong University, Shanghai, China

${ }^{3}$ Pediatric Intensive Care Unit, Shanghai Children's Medical Center Affiliated to Shanghai Jiao Tong University School of Medicine, Shanghai, China

${ }^{4}$ Department of General Surgical, Shanghai Children's Medical Center Affiliated to Shanghai Jiao Tong University School of Medicine, Shanghai, China

${ }^{5}$ Dean's Office, Shanghai Children's Medical Center Affiliated to Shanghai Jiao Tong University School of Medicine, Shanghai, China

${ }^{6}$ Evidence-Based Medicine Center, Lanzhou University School of Basic Medical Sciences, Lanzhou, Gansu, China

${ }^{7}$ Editorial Department of Journal of Shanghai Jiao Tong University (Medical Science), Shanghai Jiao Tong University, Shanghai, China

Acknowledgements Thanks to all the authors for their efforts, and thanks to $\mathrm{HL}$ for giving us strong support.

Contributors LZ, JT, YZ and HL made substantial contributions to the design of the work. WZ, QL and PN completed the search in accordance with the search strategy and screening. WL and JS extracted the data. WZ and QL assessed the quality of included documents. WL and JS analysed the outcomes. WL, JS and WZ drafted the manuscript. LZ, JT and YZ revised the manuscript. All authors approved the final submitted version of the manuscript.

Funding The authors have not declared a specific grant for this research from any funding agency in the public, commercial or not-for-profit sectors.

Competing interests None declared.

Patient consent for publication Not required.

Provenance and peer review Not commissioned; externally peer reviewed.

Data availability statement № data are available. No additional data are available.

Open access This is an open access article distributed in accordance with the Creative Commons Attribution Non Commercial (CC BY-NC 4.0) license, which permits others to distribute, remix, adapt, build upon this work non-commercially, and license their derivative works on different terms, provided the original work is properly cited, appropriate credit is given, any changes made indicated, and the use is non-commercial. See: http://creativecommons.org/licenses/by-nc/4.0/.

\section{ORCID iD}

Lie-Bin Zhao http://orcid.org/0000-0002-7000-1397

\section{REFERENCES}

1 Zhu N, Zhang D, Wang W, et al. A novel coronavirus from patients with pneumonia in China, 2019. N Engl J Med 2020;382:727-33.

2 World Health Organization. Coronavirus disease (COVID-19) pandemic. Available: https://www.who.int/emergencies/diseases/ novel-coronavirus-2019 [Accessed 27 Jul 2020].

3 World Health Organization. Novel coronavirus, 2020. Available: https://www.who.int/csr/don/12-january-2020-novel-coronaviruschina/en/ [Accessed 24 Mar 2020].

4 Vande Veegaete A, Borra V, De Buck E, et al. Guidelines of the International Federation of red cross and red crescent societies: an overview and quality appraisal using agree II. BMJ Open 2016;6:e011744.

5 Brouwers MC, Kho ME, Browman GP, et al. Agree II: advancing Guideline development, reporting and evaluation in health care. CMAJ 2010;182:E839-42.
6 Jiang M, Guan W-J, Fang Z-F, et al. A critical review of the quality of cough clinical practice guidelines. Chest 2016;150:777-88.

7 Bartko JJ. The intraclass correlation coefficient as a measure of reliability. Psychol Rep 1966;19:3-11.

8 Alonso-Coello P, Irfan A, Solà I, et al. The quality of clinical practice guidelines over the last two decades: a systematic review of guideline appraisal studies. Qual Saf Health Care 2010;19:e58.

9 Society of Pediatrics, Chinese Medical Association, Editorial Board, Chinese Journal of Pediatrics. [Recommendations for the diagnosis, prevention and control of the 2019 novel coronavirus infection in children (first interim edition)]. Zhonghua Er Ke Za Zhi 2020:58:169-74.

10 Chen ZM, JF F, SHU Q, et al. Diagnosis and treatment guideline for pediatric coronavirus disease-19 (2nd edition). J Zhejiang Univ Sci 2020:1-8.

11 HM X, Luo ZX, Xu F. Diagnosis and treatment protocol for children novel coronavirus infection (2nd edition). $J$ Pediatr Pharmacol 2020:1-8.

12 XF L, Xu Y. Experts Consensus on pediatric COVID - 19 diagnosis and treatment in Guangdong. Guangdong Med J 2020;41:217-21.

13 Pediatric Branch of Hubei Medical Association, Pediatric Medical Quality Control Center of Hubei. Recommendation for the diagnosis and treatment of novel coronavirus infection in children in Hubei (1st Editioin). Chin J Contemp Pediatr 2020;22:96-9.

14 Wang MJ, Yue SJ. Hunan neonatal medical quality control center. The programme for prevention and control of SARSCoV-2 infection in neonates in Hunan Provence. Chin Med $J$ 2020;30:65-71.

15 Wang LS, XJ H, Shi Y, et al. Perinatal and neonatal management plan for prevention and control of SARS-CoV-2 infection (2nd edition). Chin J Contemp Pediatr 2020;22:195-8.

16 Medical Association of Chinese People's Liberation Army, Preparatory Group of Pediatric Disaster. Response plans in the neonatal intensive care unit during epidemic of SARS-CoV-2 infection (2nd edition). Chin J Contemp Pediatr 2020;22:91-5.

17 Qiu YN, Li L, XY W, et al. Standardized management guideline for pediatric wards of hematology and oncology during the epidemic of corona virus disease 2019. Chin J Contemp Pediatr 2020;22:177-82.

$18 \mathrm{Fu}$ J, Zhang Y. Thoughts and recommendations on Interventional therapy of fulminant myocarditis in children with severe atrioventricular block under the situation of COVID-19. J Clin Cardiol 2020;36:209-11.

19 Bing ST, Li M, Tan L, et al. Prevention and control strategy of respiratory department in children's Hospital undergoing the COVID-19. MJCPLA 2020;32:5-9.

20 Yang LF, Dai ZY, Duan MQ, et al. Recommendations for medical staff from department of pediatrics during the treatment of 2019-nCoV infection/pneumonia. J New Med 2020;51:77-84.

21 Zhang XB, Wang CQ, Zhu QR, et al. Guiding opinions on the quality control of pediatrics in Shanghai about the prevention and control of COVID -19 (1st edition). Fudan Univ J Med Sci 2020:161-9.

22 Wei H, Chen L, Qian Y, et al. Influence of corona virus disease 2019 on mental health of children and adolescents and recommendations for family intervention. Chin J Child Health Care 2020;28:370-3.

23 Wang L, Shi Y, Xiao T, et al. Chinese expert consensus on the perinatal and neonatal management for the prevention and control of the 2019 novel coronavirus infection (first edition). Ann Trans/ Med 2020;8:47-54.

24 Shen K, Yang Y, Wang T, et al. Diagnosis, treatment, and prevention of 2019 novel coronavirus infection in children: experts' consensus statement. World J Pediatr 2020;16:223-31.

25 Chen Z-M, Fu J-F, Shu Q, et al. Diagnosis and treatment recommendations for pediatric respiratory infection caused by the 2019 novel coronavirus. World J Pediatr 2020;16:240-6.

26 Hubei Province Pediatric Medical Quality Control Center. Practice for management of pediatric ward and prevention of infecton during the dpidemic period of COVID-19. Chin J App/ Clin Pediatr 2020;02:139-42.

27 Guangdong Neonatal ICU Quality Control Center. Novel coronavirus epidemic prevention and control plan for neonatal unit in Guangdong Province. J Pract Med 2020;36:1121-4.

28 Xing Y, Zhang J, Han T, et al. Exploration of management strategies about novel coronavirus pneumonia in pediatrics department of General Hospital. J Peking Univ 2020:1-11.

29 Jiang Y, XX L, Jing MR, et al. Expert consensus on novel coronavirus infection diagnosis, treatment and prevention (second edition). Chin $J$ Pract Pediatrics 2020;02:143-50.

30 Lv Z, AM L, Zhang NX, et al. Strategies for COVID-19 prevention and control in children's specialist hospitals. J Clin Pediatrics 2020;38:313-6. 
$31 \mathrm{HZ}$ X, Chen SH, JF F, et al. Recommendation on novel coronavirus infection prevention and control for children. Chin J Hosp Manage 2020;04:316-9.

32 Zhou YS, Ma X, Guo SB, et al. Study on ward division and classification management of prevention and control of COVID 2019 infection for the perinatal - neonates in comprehensive hospital during the epidemic. Chin J Crit Care 2020;40:395-8.

33 Jiangsu Province expert group of novel coronavirus pneumonia medical treatment. Expert recommendation of novel coronavirus infection diagnosis and treatment for children in Jiangsu Province (trial version 1). J Nanjing Med Univ 2020;40:309-14.

34 Shen $\mathrm{Q}$, Wang $\mathrm{M}$, Che $\mathrm{R}$, et al. Consensus recommendations for the care of children receiving chronic dialysis in association with the COVID-19 epidemic. Pediatr Nephrol 2020;35:1351-7.

35 Carvalho WBde, Gibelli MABC, Krebs VLJ, et al. Expert recommendations for the care of newborns of mothers with COVID-19. Clinics 2020;75:e1932.

36 Calvo C, López-Hortelano MG, Vicente JCdeC, et al. Recommendations on the clinical management of the COVID-19 infection by the «new coronavirus» SARS-CoV2. Spanish Paediatric Association working group. Anales de Pediatría 2020;92:241.e1-241.e11.

37 Chorney SR, Elden LM, Giordano T, et al. Algorithm-Based pediatric otolaryngology management during the COVID-19 global pandemic: a children's Hospital of Philadelphia clinical consensus. Otolaryngol Head Neck Surg 2020;163:25-37.

38 Trevisanuto D, Moschino L, Doglioni N, et al. Neonatal resuscitation where the mother has a suspected or confirmed novel coronavirus (SARS-CoV-2) infection: suggestion for a pragmatic action plan. Neonatology 2020;117:133-40.

39 Gray B, Semsarian C, Fatkin D, et al. Patients with genetic heart disease and COVID-19: a cardiac Society of Australia and New Zealand (CSANZ) consensus statement. Heart Lung Circ 2020:30134-7.

40 Bann DV, Patel VA, Saadi R, et al. Best practice recommendations for pediatric otolaryngology during the COVID-19 pandemic. Otolaryngol Head Neck Surg 2020;162:783-94.

41 Leboulanger N, Sagardoy T, Akkari M, et al. COVID-19 and ENT pediatric otolaryngology during the COVID-19 pandemic. guidelines of the French association of pediatric otorhinolaryngology (AFOP) and French Society of otorhinolaryngology (SFORL). Eur Ann Otorhinolaryngol Head Neck Dis 2020;137:177-81.

42 Ministry of health. Interim guidance for breastfeeding promotion during COVID-19 pandemic. Available: https://www.moh.gov.sa/
Ministry/MediaCenter/Publications/Documents/Interim-GuidanceFinal.pdf [Accessed 30 May 2020].

43 Klein JD, Koletzko B, El-Shabrawi MH, et al. Promoting and supporting children's health and healthcare during COVID-19 International Paediatric Association Position Statement. Arch Dis Child 2020;105:620-4.

44 Chen Y, Hu S, Wu L, et al. Clinical practice guidelines for hypertension in China: a systematic review of the methodological quality. BMJ Open 2015;5:e008099.

45 Wong ELY, Wong SYS, Lee N, et al. Healthcare workers' duty concerns of working in the isolation ward during the novel $\mathrm{H} 1 \mathrm{~N} 1$ pandemic. J Clin Nurs 2012;21:1466-75.

$46 \mathrm{Yu}$ IT, Xie ZH, Tsoi KK, et al. Why did outbreaks of severe acute respiratory syndrome occur in some hospital wards but not in others? Clin Infect Dis 2007;44:1017-25.

47 Wong SCY, Kwong RT-S, Wu TC, et al. Risk of nosocomial transmission of coronavirus disease 2019: an experience in a general ward setting in Hong Kong. J Hosp Infect 2020;105:119-27.

48 Gogle J. Using simulation-based learning to prepare for a potential cardiac emergency on the labor unit. Nurs Womens Health 2017;21:20-7.

49 World Health Organization. Clinical management of COVID-19: interim guidance, 2020.

50 British Association of Perinatal Medicine (BAPM). British association of perinatal medicine resource page on COVID-19. Available: https:// www.bapm.org/pages/182-perinatalcovid-19-resources [Accessed 30 May 2020].

51 American Academy of Pediatrics. Resource page on COVID-19. Available: https://services.aap.org/en/pages/2019-novel-coronaviruscovid-19-infections/ [Accessed 30 May 2020].

52 UNICEF. Coronavirus disease (COVID-19): what parents should know. Available: https://www.unicef.org/stories/novel-coronavirusoutbreak-what-parents-should-know [Accessed 24 May 2020].

53 Center for Disease Control and Prevention. Pregnancy \& Breastfeeding. Information about Coronavirus Disease, 2019 Available: https:// www.cdc.gov/coronavirus/2019-ncov/specificgroups/pregnancy-guidance-breastfeeding.html [Accessed 30 May 2020].

54 Bapistella S, Hamprecht K, Thomas W, et al. Short-Term pasteurization of breast milk to prevent postnatal cytomegalovirus transmission in very preterm infants. Clin Infect Dis 2019;69:438-44.

55 Moher D, Liberati A, Tetzlaff J, et al. Preferred reporting items for systematic reviews and meta-analyses: the PRISMA statement. PLoS Med 2009;6:e1000097. 